\title{
25 Research Soure \\ Out-of-clinic and Self-managed Abortion in Bangladesh: A Qualitative Study of Menstrual Regulation Providers
}

Bonnie Crouthamel ( $\sim$ bcroutha@gmail.com )

University of California San Diego https://orcid.org/0000-0003-0341-0083

Erin Pearson

IPAS

Sarah Tilford

University of California San Diego

Samantha Hurst

University of California San Diego

Dipika Paul

IPAS

Fahima Aqtar

IPAS

Jay Silverman

University of California San Diego

Sarah Averbach

University of California San Diego

Research article

Keywords: Abortion, Menstrual regulation, Family planning

Posted Date: October 7th, 2020

DOI: https://doi.org/10.21203/rs.3.rs-79990/v1

License: (a) (i) This work is licensed under a Creative Commons Attribution 4.0 International License. Read Full License 


\section{Abstract}

Background: In Bangladesh, abortion is illegal except to save a woman's life, though menstrual regulation (MR) is permitted. MR involves the use of manual uterine aspiration or Misoprostol (with or without Mifepristone) to induce menstruation up to 10-12 weeks from the last menstrual period. Despite the availability of safe and legal MR services, abortions occur outside of the legal system and are associated with high complication rates.

Methods: Qualitative semi-structured interviews (SSIs) were conducted with 25 trained MR providers from urban tertiary care facilities in six different cities in Bangladesh. SSIs explored providers' attitudes about the practice of unsanctioned MR/abortion providers and self-managed MR/abortion, their experiences treating patients who sought abortion services out-of-clinic, barriers/facilitators to safe MR/abortion, and MR/abortion definitions.

Results: There were three predominant yet overlapping themes: (i) logistics of obtaining MR/PAC/abortion, (ii) provider attitudes, and (iii) overcoming barriers to safe MR. Lack of consensus among trained providers revealed challenges for defining MR/abortion and gestational age cutoffs. Increasing post-abortion care (PAC) services may be due to patients purchasing Mifepristone/Misoprostol from pharmacists who do not provide adequate instruction, or patients may be directed to untrained providers by brokers, who intercept patients entering the hospitals/clinics and receive a commission from unsanctioned clinics for bringing patients. Perceived barriers to accessing in-clinic MR include distance, family influence, brokers, lack of knowledge, and provider attitudes toward MR.

Conclusions: Providers in urban tertiary care facilities in Bangladesh see primarily the complicated MR/PAC cases, and the safety of out-of-clinic/self-managed abortion is unknown. Lack of standardization among providers of MR definition/gestational age cutoffs may affect patient care and MR access. MR safety may be improved by eliminating brokers. A harm reduction approach to improve MR/abortion care provided by pharmacies may improve safety and access. Policy makers should consider increasing training of frontline health workers, such as Family Welfare Visitors (FWVs) to provide evidence-based information about Mifepristone/Misoprostol.

\section{Background}

Unsafe abortion is one of the leading causes of maternal mortality worldwide, and maternal deaths disproportionately occur where abortion is restricted or illegal. ${ }^{1}$ In Bangladesh, abortion is illegal except to save a woman's life. However, menstrual regulation (MR) has been available as a method of family planning in the country since $1979 .{ }^{2}$ MR involves the use of either manual vacuum aspiration (MVA) or medications (Misoprostol with or without Mifepristone) without definitive diagnosis of pregnancy to induce menstruation and can be performed up to 12 weeks from the last menstrual period for doctors and 10 weeks for nurses. ${ }^{2}$ Menstrual regulation is sanctioned by the government as a family planning method rather than an abortion, and safe MR services are offered within government and private health facilities. ${ }^{3}$

Despite this formal system that allows for abortion care in a clinic, many women continue to obtain abortions outside of this system. Some of these women eventually present to clinics for post-abortion care (PAC). In 2010, approximately 231,400 women were treated for complications of out-of-clinic abortion, and this is estimated to be only $40 \%$ of the total complications resulting from out-of-clinic abortion. ${ }^{4,5}$ In a study utilizing surveillance 
data from more than 100,000 pregnancies in Bangladesh, MR was found to have a lower risk of maternal mortality than live birth, while out-of-clinic abortion had a higher maternal mortality rate than live birth. ${ }^{6}$ In this study, methods of abortion outside the health system consisted of using medications or receiving a procedure from an informal provider. It was unknown which medications were used, or if women were purchasing these medications over the counter from pharmacies to self-induce abortion ${ }^{6}$.

A 2012 survey of healthcare facilities and providers identified that younger women with less education are more likely to have an out-of-clinic abortion in Bangladesh ${ }^{5}$. Systemic barriers to accessing formal MR services include provider shortages in facilities expected to provide MR, lack of equipment, or provider religious or cultural

opposition. ${ }^{5}$ Additionally, about $26 \%$ of women are turned away from MR facilities, most often due to being beyond the legal gestational age limit. ${ }^{3}$

The government health system and various NGOs in the country have well-regulated MR clinics that provide safe care with few complications. However, due to the acceptability and accessibility of pharmacies and other informal facilities, women often do not use these well-regulated clinics as the first point of access for MR care ${ }^{7}$. Centralized health clinics are often seen as being more costly and difficult to access, while close-to-community providers are more familiar and trustworthy ${ }^{7}$.

Although surveys and cross-sectional studies of MR providers have been performed, qualitative studies among trained MR providers are lacking. Qualitative assessments have the potential to lend more in-depth insight into provider experiences with women who have sought out-of-clinic abortions and their perspectives on barriers to safe abortion care. Interviewing providers can also reveal systemic barriers that might not be readily apparent to patients and other stakeholders. The aim of this study is to explore and identify systemic, community-related, and/or cultural barriers and facilitators that may impact the incidence of safe MR, methods of self-induced abortion, and abortion care received prior to presenting for MR/PAC via qualitative semi-structured interviews with trained MR providers in tertiary care facilities. These provider perspectives will help develop recommendations for culturally appropriate interventions that may decrease barriers and increase access to safe abortion care for women in Bangladesh.

\section{Methods}

\section{Setting}

MR providers, consisting of doctors and nurses, were recruited from six medical college hospitals and their attached family planning clinics, each in a different divisional (regional) capital of Bangladesh. The NGO-run RHSTEP (Reproductive Health Services Training and Education Program) clinics are well equipped to provide menstrual regulation, post abortion care, and contraception and their clinical services have been monitored and evaluated by Ipas. Ipas is an NGO that works to ensure safe provision of abortion care globally including through local partnerships in Bangladesh. Each clinic is attached to an associated medical college hospital, which are tertiary care facilities.

\section{$\underline{\text { Recruitment }}$}


Purposive sampling was used to recruit providers from different practice settings to obtain a diverse range of experiences with patients who attempted out-of-clinic and self-induced MR/abortion. Therefore, doctors and nurses from both RHSTEP clinics and medical college hospitals who provide MR and PAC services were recruited by study staff at regional meetings. During these meetings, all providers and counsellors working in the selected RHSTEP clinic and medical college hospital were invited to participate in the study. Providers interested in participating voluntarily signed up for the study at the conclusion of these meetings. Inclusion criteria for the interviews were that the providers had to work at one of the six RHSTEP clinics or attached medical college hospitals and provide MR and PAC services. Providers were contacted to schedule the qualitative interview. Prior to the interview, informed consent was obtained by the interviewer. Qualitative interviews were conducted in a private location. Providers who completed the interviews were compensated for their pariticipation (the equivalent of \$3 US according to local standards).

\section{Qualitative Interviews}

Local Bangladeshi qualitative interviewers were trained to conduct the interviews in Bangla at a private location in the provider's workplace. In-depth interviews were semi-structured, lasted approximately 1 hour, and assessed providers' knowledge of MR and abortion in Bangladesh, knowledge/experience with unsanctioned MR providers, knowledge/experience with patients attempting self-induced abortion, barriers and facilitators to safe abortion, and personal attitudes and moral perspectives of MR/abortion in general. The questionnaire used in this study was developed specifically for this study and was piloted among five participants before data collection began (Additional File 1). All semi-structured interviews were audio recorded, transcribed, and translated from Bangla to English by trained research study staff in Bangladesh. Transcript file names did not include personal identifiers, but were labeled with unique integer and character identifiers that were created by in-country research staff. All audio and text files remain stored in a secured multi-file location at the Ipas office in Dhaka, Bangladesh. Only English version transcripts were used for the team-based qualitative analysis that was lead by the study PI in the United States. Ethical approval of all study instruments and protocols was granted by the Bangladesh Medical Research Council (BMRC) and the University of California, San Diego Institutional Review Board. Quotes are non-identifiable and labelled with participant location, practice setting (RHSTEP vs. medical college hospital), and role (doctor vs. nurse).

\section{Data Analysis}

A directed content analysis approach was used to promote a structured analytical procedure for reviewing participant transcripts ${ }^{8}$. A qualitative researcher began the initial cycle of open coding, making use of the interview guide to identify contextualized segments of data based on the interview questions ${ }^{9}$. Novel codes were created for data that could not be assigned using the question-based coding schema ${ }^{10}$. A team-based approach using three coders was used for the second coding cycle to resolve discrepancies in assignment or description of codes by discussion and consensus among members of the research staff ${ }^{11}$. Interview transcripts were uploaded along with the codebook to Dedoose ${ }^{12}$ (Version 8.0.42, 2019), a web-based qualitative analysis program. This program has flexible features to facilitate organizing and sorting the transcripts, as well excerpting quotes, and locating key words and phrases responding to the original interview guide. Using Dedoose and the final draft codebook, a third cycle of review assisted in merging some of the earlier excerpts 
and into nested categories because of conceptual similarity or redundancy, while other codes were dropped due to lack of utility to the specific aims of the study. At the conclusion of coding, we extracted all of the coded excerpts and refined their organization by framing the coding patterns, interconnections and overarching themes in the study data. Thematic saturation was achieved.

\section{Results}

A total of 25 trained MR providers were recruited from medical college hospitals and RHSTEP clinics, and all consented to participate in the semi-structured interviews. Twelve nurses and 13 doctors participated. There were 19 providers from medical college hospitals and 6 providers from RHSTEP clinics.

There were three predominant yet overlapping themes that providers used to discuss MR, PAC and abortion care in Bangladesh:

i. Logistics of MR/PAC/abortion in Bangladesh: MR providers described the logistics of obtaining MR, PAC, and abortion in Bangladesh and defined each of the terms, gestational age cutoffs and who is providing them. They detailed their experiences treating patients that have sought out-of-clinic and self-managed abortion.

ii. Attitudes: MR providers detailed their own attitudes about MR in general, unsanctioned MR providers (including pharmacists), and self-management of abortion.

iii. Overcoming Barriers: MR providers outlined perceived barriers to clinic MR (and facilitators to out-of-clinic $\mathrm{MR}$ ) and gave recommendations for interventions to overcome those barriers.

Interwoven among these themes were the impact of various factors on patient autonomy when deciding to have MR or abortion.

\section{Logistics of MR, PAC, and abortion in Bangladesh}

\section{Definitions and gestational age cutoffs}

Abortion is a complicated word with many meanings, and there was a lack of consistency among trained MR providers about the definition of MR, abortion, legality, and appropriate or legal gestational age cutoffs for receiving MR care. This lack of consistency was present among all sites, practice settings, and provider roles. Some providers defined the difference between MR and abortion based on the viability of the pregnancy-MR being provided only for viable pregnancies and abortion for non-viable pregnancies or in the setting of pregnancy loss with retained tissue. There were varying opinions as to whether abortion could be induced. One nurse stated, "MR is.... when a patient decides to terminate their child... while abortion happens when it has already aborted but [is] not properly cleaned" (Nurse 10, medical college hospital). Another nurse agreed that "abortion is not a deliberate process, but MR is a deliberate process" (Nurse 4, medical college hospital). However, other providers acknowledged that though "abortion" refers to a non-viable pregnancy, it can be induced:

There is no bleeding in case of MR, she only wants to do termination. However, in case of abortion...she does not want to take this baby and take medicine, sometimes...the product is not released properly (Doctor 13, medical 
college hospital).

Others agreed that abortion is induced because it is done with knowledge of a pregnancy, whereas MR is done if the woman does not know she is pregnant:

MR is...if menstruation does not occur without being sure about pregnancy, then if the procedure is done that is called MR. But if it is done by knowing about the pregnancy then of course that is abortion (Doctor 4, medical college hospital).

With regards to legality and gestational age cutoffs, there was no standardization as to how far along MR or abortion could be performed or if abortion can actually be performed in Bangladesh:

Actually there is no abortion in Bangladesh...Some patients tell straightway, "I have come here for abortion." Then we ask them how many months her period is stopped? Suppose she replies, 2 or $2 \frac{1}{2}$ months, then we make her correct that she has come for regularizing her menstruation (Nurse 2, RHSTEP).

In contrast, another doctor stated, "In Bangladesh we are doing abortion...those who are under 12 weeks, we do MR services to them" (Doctor 11, medical college hospital). Several providers acknowledged that MR can be performed up to 10-12 weeks depending on the type of provider, but there were many deviations from this standard. According to one nurse, "Abortion can be done within 35 weeks...MR can take place only within 14 weeks" (Nurse 8, medical college hospital). Another nurse stated, "Abortion can happen in 8 weeks or 12 weeks and even in 14 weeks but you have to do MR within 8 to 10 weeks. If it is 12 weeks, then it is risky" (Nurse 10, medical college hospital). One doctor said, "[MR can be done before] seven menstrual weeks" (Doctor 6, medical college hospital). One RHSTEP doctor stated, "MR is generally 10 weeks for doctors and 8 weeks for nurses" (Doctor 8, RHSTEP). If providers are unaware of the legal gestational age cutoffs for MR, it may contribute to patients begin turned away from facilities, even if they are within the legal gestational age limit.

\section{Informal out-of-clinic providers}

According to providers, patients sometimes are referred to informal out-of-clinic providers by brokers and via word-of-mouth from family and community members. Brokers intercept patients entering the hospitals/clinics for MR or PAC and receive a commission from informal clinics for bringing patients. Although some traditional healers and homeopathic providers perform out-of-clinic abortions, a lot of the informal providers have previously worked in the hospitals and sanctioned clinics as observing assistants but have not usually received formal training:

These service providers may have some experience, for example, this aunty has become an expert by watching MR regularly here. Like her, these service providers might have worked for a center and then they decide to open a private clinic by their own (Doctor 13 , medical college hospital).

Do you know that some of our Ayas [sisters/hospital workers], they introduce themselves as Nurse to the patients and take them? But they are not nurse, they do not have any training (Nurse 6, medical college hospital). 
The settings in which these informal providers practice were described as unhygienic without proper sterilizing equipment, often located in the provider's private home. One doctor explained, "We do autoclave regularly our instrument. We don't use any instrument twice without autoclave. But those service providers don't do that. They wash the instruments with water and use it again and again" (Doctor 3, RHSTEP).

The methods used to induce MR/abortion can vary by the type of provider. Traditional healers tend to use herbs and tree roots, though providers report this is becoming less common:

Previously we got many patients who have been maltreated by the traditional healers. They used herbs and indigenous methods...Nowadays these types of clients has reduced...Some patients arrived [at the] hospital with septic infections and herbal roots inserted into their uterus (Nurse 2, RHSTEP).

More commonly reported was use of MVA, as "MVA set is available to purchase... They perform with the instruments in this way" (Doctor 4, medical college hospital). Some informal providers perform abortion procedures "by hand-curette" (Nurse 3, medical college hospital).

Providers at RHSTEP and in the medical college hospitals come to know of these informal providers because they treat patients who have complications, which creates unfavorable opinions of the informal providers. They describe that some patients come after seeing unsanctioned providers with "...septicemia, septic abortion, incomplete abortion...Today, we had a patient [in which] part of the digestion system was cut off during MR" (Doctor 2, medical college hospital). Other providers acknowledge that some out-of-clinic MR could be performed safely, as "those who get performed without complications, they do not come to us. They go back from there. Those who face complications they come to us" (Doctor 4, medical college hospital).

\section{Self-managed abortion}

With regards to self-management of abortion, providers indicate that although traditional methods provided by herbalists and traditional healers are still being used, using Misoprostol (with or without Mifepristone) is being more commonly reported, which is often purchased over-the-counter in pharmacies. Patients obtain various methods "through others, friends and relatives and also from the brokers.... The family members instruct them. Husband goes to the medicine shop and told the drug-seller that his wife missed her period" (Nurse 1, medical college hospital). Traditional methods include herbs and "medicine made from grass root. [One patient] put this medicine in cervix...she poked [back and forth] with it in the uterus. Many people think that if they put pressure in abdomen fetus can expel" (Doctor 5, RHSTEP). Often, providers described patients trying several different methods to self-manage abortion before finally presenting to a formal clinic, though one commonly reported method was medications purchased over the counter from pharamacies without a prescription. The providers also noticed a change in the number and type of services they provide due to these practices:

Some take medicine; some go to the sisters. In [many] cases those who come to us have tried all these methods.

First they take homeopathic, then go to the kabiraj (herbalist) and go to the sisters. When it becomes incomplete, then they come to us (Doctor 7, medical college hospital).

MR services [are] decreased. You can get misoprostol pills at pharmacy and everyone is using it... They do not need anything to buy it from pharmacy. Pharmacy gives the medicine to everyone like to the patients or to the 
husbands (Doctor 5, RHSTEP).

Providers are aware of self-management practices because they treat patients with complications which creates unfavorable attitudes about self-management. Complications from self-management include "incomplete [abortion and] excessive bleeding...Another problem is that even though they do it, the pregnancy continues. They say that they had taken medicine but it continued [to] 16-18 weeks" (Doctor 9, medical college hospital). Another provider encountered a patient "with ectopic pregnancy! She came here after taking medicine advised by her neighbor. But, she needed an open surgery... Some of them who are taking medicine from the pharmacists are having incomplete abortion" (Nurse 2, RHSTEP). Many complications from pharmacies are thought to be due to inadequate screening and instruction:

The MR through medicine also needs doctor's instructions. We have to tell them about the dose, its use, through which route it would pass, the complications that may arise, full warning signs. They will not get such instructions from the pharmacy (Doctor 10, RHSTEP).

Other providers acknowledge that, again, they are only seeing the complications from self-managed abortion, and other cases may be happening safely. One provider knows "about two to three persons who have done it by themselves without any problem. It was complete" (Doctor 10, RHSTEP).

\section{Spectrum of provider attitudes}

\section{MR in general}

There were a variety of attitudes among trained MR providers with regards to the ethics and practice of MR, and ethics was often tied to religion. Providers working for RHSTEP tended to have a more positive view of MR, explaining the value of MR services and the benefit to patients. They also tended to respect patient autonomy and did not change practice based on the patient's situation. One RHSTEP provider explained, "[MR] is useful for [many] girls. Otherwise many girls had to commit suicide or suffer a lot" (Nurse 2, RHSTEP). Some providers in medical college hospitals also had positive views and respected patient autonomy in decision making:

From religious point of view I had some obligations. But when I got the training of PAC and MR from IPAS, my view had changed. That training was positive for me. Now I always provide this service to the patients (Doctor 1 , medical college hospital).

[I tell patients when deciding on MR] Even husband's confirmation is not needed; your right is the main. You have to do which is right for you. You have to think about your health, your in laws will not think about it (Nurse 11, medical college hospital).

However, when providers have negative attitudes about MR or impose their own moral judgement on the patient's decision, patient autonomy can be affected, creating barriers to MR. Some providers had religious hesitations to providing MR, and others would only perform MR in certain situations:

Usually we do not try to give MR and PAC services to the patients. First of all, we see how many children they have, how much her demand is... If we see her child age is one year or 8/9 months old then we think about MR 
and PAC services for her because these are good for her as her child is very much young for taking another child (Nurse 7, medical college hospital).

I think that one should not go for a MR. This would be advantageous for all. We are Muslims. It is not the question of a Muslim only, in fact, every religion forbids killing a life (Nurse 8, medical college hospital).

Other providers will only perform MR "if there is husband, mother-in-law, or mother with them" (Doctor 7, medical college hospital). If providers refuse to perform MR, this creates barriers, causing women to seek care from other informal sources. According to one nurse:

She has to do an abortion anyhow. It can be by doctor or nurse. But sometimes doctors or nurses do not give her any counseling and blame her to be pregnant because she is unmarried and ignore her. That time she goes to inexpert midwife or quack (Nurse 10, medical college hospital).

\section{Patients seeking MR}

Providers sometimes judged patients for their decisions, especially if they sought an out-of-clinic abortion. One provider described punitive behavior toward patients presenting with complications from out-of-clinic abortion:

We then tell them, "Why have you delayed? Why have you done this?"...They need blood but will not come to hospital. "Now you have come when you are about to die." We rebuke them like this when they come with problem (Nurse 6, medical college hospital).

Other providers judged patients who seek MR in general as having lower education, affecting how providers view their decision making capacity. This was common throughout all provider roles and practice settings. One doctor explained, "Educated people are not much to come for MR, they are conscious. MR services take place around mostly lower class" (Doctor 3, RHSTEP). Another provider thought lower class women had different motivations than other women, stating, "Women do not want to destroy [their] baby - those who want it - it is done by the slum dwelling women... They want to do it. But directly no woman wants to destroy her baby" (Nurse 1, medical college hospital).

\section{Overcoming barriers to safe MR}

\section{Identifying barriers}

Providers identified several barriers to obtaining in-clinic MR care, including logistical, cultural, provider/health facility related barriers, and brokers. Logistical barriers include cost, lack of knowledge and family obligations. When seeking MR, "the hospitals seem distant for [the clients], and they think about the expense that will occur because of the distance [so] they get a sister beside them who does the MR" (Doctor 1, medical college hospital). Furthermore, clients "may not be aware of service. As you know sister, in many villages these messages have not reached yet" (Nurse 6, medical college hospital). With regards to family, husbands and mothers-in-law often dictate the care that a woman receives, and family obligations require the woman to stay close to the home. 
Opinions of the family can affect patient autonomy. One nurse spoke of the consequences of disobeying the mother-in-law:

Some in-laws say like “Do not go there. It will work out if you take herbs, if you drink holy water..." Then they will go to the religious leader [and] traditional healers. When they come they say that their mothers-in-law were creating obstructions for them...[but] if they do not obey their mothers-in-law...maybe they will be divorced, they will be thrown out of their house, their in-laws will arrange another marriage (Nurse 6, medical college hospital).

Another doctor discussed that women's family obligations make it difficult to be away for longer periods of time:

Many feel that coming to the hospital means staying here for $2 / 3$ days...If someone gets service in her locality from a quack doctor or a herbal doctor, she can then avail it...In addition, if she gets anything done in the locality, she can also look after her family (Doctor 10, RHSTEP).

Additionally, religion can create a cultural barrier to accessing in-clinic MR, though there were varying opinions on the ethics of MR in the context of the Muslim religion. In certain situations, some providers unequivocally stated that "There are religious obstacles...especially the Muslims face religious obstacle" (Nurse 11, medical college hospital). However, one doctor in Dhaka disagreed:

If there are religious reasons they will not be coming to centre for MR. Even the wife of a Huzur [Muslim religious leader] is also coming to us for MR as well as the wives of a Moulana or Imam...It is not the religious influence (Doctor 1, medical college hospital).

Other providers indicated that religious opinions of MR may not be homogeneous, varying with different levels of knowledge and conservativism:

There are some types of people but they are not real huzur. Who are real huzur, they understand these issues, and they are conscious persons...However, who are fanatical they think my wife would not go there [for MR] (Nurse 7, medical college hospital).

Regardless, it seems like religion can be a barrier for women in certain circumstances.

Furthermore, providers discussed that there are aspects of the health centers and the providers themselves that create barriers to in-clinic MR. Time constraints and provider attitudes cause patients to seek care elsewhere, especially in government health facilities:

One physicians supposed to see 200 - 300 patients within 6 hours. The same physician...in a private clinic, he has to see 5 - 6 . He gives 20 minutes to 30 minutes to each patients in a private clinic...In [other] centers many patients come for service but the providers don't provide services at all (Nurse 2, RHSTEP).

Providers also recognize that their own behavior can deter patients from coming to health centers. One provider explained, “We doctors don't want to do counselling. Because of this the patients don't understand what has happened to them... we also cannot explain properly due to lack of time. There is a gap here" (Doctor 8, RHSTEP).

Overwhelmingly, providers of all roles from all different practice settings and sites indicated that brokers create immense barriers for patients in seeking in-clinic MR. Brokers intercept patients seeking MR from RHSTEP or the 
government health facility and take them to other clinics, which are often unsanctioned, where they receive a commission:

They maintain a strong syndicate from the main gate of the hospital... They convince patient to go the private clinic to receive better quality medical service compare to government hospital. The service receivers go to the private clinic and take treatment facilities from [a] quack (Doctor 12, RHSTEP).

Brokers not only stand outside the hospital gate, but can be members of the hospital staff as well, charging patients for referrals. One nurse said that "the maids, ward boys, they are brokers...they are taking one thousand taka from [patients]" (Nurse 12, medical college hospital). Since they may be members of hospital staff and health centers can be large and difficult to navigate, patients often trust the brokers to take them to the correct location, though they end up in informal clinics. Even if patients become suspicious of brokers, once they are in another informal clinic, they sometimes have to pay just to leave:

[One woman] was searching for RHSTEP clinic. A broker took her to another clinic. Seeing the environment...the patient assumed that something is wrong. She told that broker, "...I don't want to stay here, I want to go back." The broker then said, “...You have to pay before you leave otherwise we won't let you leave” (Nurse 2, RHSTEP).

In this case, the broker created a barrier to in-clinic MR and affected the woman's autonomy since she was not taken to her choice of provier.

\section{How to improve MR care}

When discussing how MR care can be improved in Bangladesh, providers suggested further education/outreach, training, increasing services in more peripheral settings, and punitive action against brokers. Some providers recommended education of villagers about MR services via meetings and leaflets. Although some advocated for punishing untrained providers, others advocated for further training, stating "It would be better if those [untrained providers] could actually be identified. And it would be best if they could be provided with training...As you know, sisters can be trained" (Doctor 4, medical college hospital). One nurse also suggested training providers in appropriate behavior:

When you do adequate counselling then the patient will go and tell that [this provider] is so good, she spoke to me nicely. Behaviour is also very good...and she would speak well of us. She would go back and propagate (Nurse 5, medical college hospital).

Although there are some providers doing MR in more rural settings, many providers thought that care outside of the tertiary care centers could be improved and expanded to include community based trained frontline health workers called family welfare visitors (FWV):

There are also some trained nurses who can do this kind of service. They are also working in periphery. We can trained them more and send them to the sub centres. Even we can train the FWV. At least they have some knowledge and they will act accordingly (Doctor 1, medical college hospital).

Finally, many providers wanted punitive action against the brokers: 
If any journalist would help [with the brokers], investigate this issue placing self as a patient, then these criminals would have been exposed...These people are harassing these MR patients, this should be stopped (Nurse 2, RHSTEP).

\section{Discussion}

Many women in Bangladesh seek MR services outside of formal clinical settings, whether it is from other providers or self-management of abortion. Although women still visit informal providers and traditional healers, who frequently use herbs, foreign objects in the cervix/uterus, or MVA or sharp curette, MR providers thought that the practice of taking medications provided by pharmacies was becoming more common. Complications encountered by providers in tertiary care settings include bleeding, infection, retained products of conception, failure to end pregnancy, uterine perforation, and sometimes death, causing most providers to have negative attitudes about informal providers and pharmacists offering medication abortion. Other barriers to clinic MR cited by providers included cost, distance, lack of knowledge of services, family obstruction, religion, and provider behavior. The overwhelming majority of providers especially emphasized that brokers are a barrier to clinic MR care and create unsafe care for patients. Despite interviewing trained MR providers, there was a lack of consistency about the definitions of MR and abortion as well as gestational age cutoffs for performing MR legally.

The strengths of this qualitative study are that it outlines a subjective and experienced view of out-of-clinic and self-managed abortion from the perspective of trained MR providers in tertiary care settings who routinely treat both MR/PAC patients and complications from out-of-clinic abortion. The perspectives of trained MR providers in this study are consistent with several other studies on MR/abortion in Bangladesh. Similar to other studies, the providers in our study described that complications of out-of-clinic abortion are still higher than in-clinic MR, though the use of less safe methods, such as foreign objects in the cervix/uterus and sharp curette are decreasing over time $3,4,6,13$. The description of pharmacists providing inadequate instruction when prescribing Mifepristone and Misoprostol is also consistent with prior studies ${ }^{14}$. Footman et al. followed MR clients seeking medications from pharmacies and found that $40.4 \%$ of clients received no instruction, and only $8.7 \%$ received written information or pictures ${ }^{15}$. During mystery client visits to pharmacies, Huda et al. found that only $54 \%$ of pharmacists provided the recommended dosage of Mifepristone and Misoprostol, and only $11 \%$ provided any counseling on possible complications ${ }^{16}$. A harm reduction approach to improve the quality of care provided in pharmacies may improve safety of self-managed abortion.

Other studies have also cited cost, distance, lack of knowledge of services, family obligations, long wait times at government hospitals, and perceived lack of quality of care at hospitals as creating barriers or delays in obtaining MR in sanctioned clinics ${ }^{3,7}$. They have also described similar facilitating factors of pharmacies and close-to-community providers, such as less cost and less of a need for transport, when seeking MR services ${ }^{7}$. When discussing how MR services can be improved, MR clients have agreed with the viewpoints of the providers in this study that better instruction and treatment of patients needs to be provided, and services need to be more readily available in peripheral settings ${ }^{17}$. The Ministry of Health in Bangladesh has prioritized increasing the number and responsibilities of frontline health workers, such as FWVs, to supplement family planning care in more rural areas ${ }^{18}$. Expanding the scope of FWVs to include MR with Mifepristone and/or Misoprostol may improve out-of-clinic abortion safety. 
One finding of this study was that provider attitudes may affect access to MR care and undermine patient autonomy. The definition of quality contraceptive counseling articulated by Judith Bruce in 1990 not only respects patient dignity and autonomy, but also increases access to family planning, and recent revisions have promoted expanding this model to all aspects of reproductive health ${ }^{19}$. Expanding these guidelines to MR services in Bangladesh may also increase access to safe MR services for patients. Additionally, as selfmanagement via medications from pharmacies is becoming more common, training providers to support patients by providing compassionate post-abortion care rather than punitive behavior toward patients for their choices may further facilitate patients seeking care in the future or referring others in their communities. Studies have previously mentioned that although certain providers are trained in MR, they may not be practicing MR due to their personal or religious beliefs ${ }^{20}$. The providers in this study alluded to certain scenarios in which they would not perform MR or would try to encourage a patient to not seek MR, such as when a husband/mother-inlaw were not present or if a married patient did not yet have children. The issue of religion was complex, with some providers saying the religion was a barrier for Muslim patients, while others said that they have treated the wives of Imams, and religion was not a barrier. This complexity in opinions may be explained by varying levels of conservativism in religion. One limitation of the study is that the providers' own religious beliefs and level of conservativism was not assessed, rather they were asked to comment on their experiences treating patients. Providers commonly described patient receiving MR as being of a lower class or less educated, which may also affect how they view clients' ability to make autonomous decisions about their pregnancy intention.

Although previous studies have described that patients are turned away from MR facilities, the main reason described in Hossain et al. was not provider attitudes but being beyond the legal gestational age limit ${ }^{3}$. In that study, most women thought they were 3-4 months pregnant at the time of seeking MR, though the exact gestational ages are unknown, and the most common gestational age cutoff for MR given was 12 weeks ${ }^{3}$. A surprising finding of this study was the lack of standardization among trained MR providers of the definitions of MR and abortion as well as gestational age cutoffs for performing MR legally. Officially, doctors can perform MR until 12 weeks, and mid-level providers can perform MR until 10 weeks ${ }^{2}$. However, the varying gestational age cutoffs could not be described by different provider cadres, as the reported range in which MR could be performed varied between 7 and 14 weeks. This discrepancy persisted among all provider roles, all practice settings, even in certain RHSTEP clinics, whose providers have been formally trained by Ipas. In the medical college hospitals, providers may not have received formal Ipas training and may have been trained through the government medical education system, explaining some of the discrepancy. In Hossain et al., most women were not aware of the legal gestational age cutoff for $\mathrm{MR}^{3}$. If there is no standardization of gestational age cutoffs among trained MR providers, it may make it difficult to provide education about legal MR cutoffs, and patients may be turned away even if they are within the legal MR window. This study highlights that further training of MR providers is needed.

Furthermore, one barrier to clinic MR (and safe MR in general) that was repeatedly highlighted by providers in this study was the presence of brokers at medical college hospitals and RHSTEP clinics. Brokers have been briefly mentioned in other studies, but the fact that most providers from all clinical settings and all provider roles described their detriment to patient care was a novel finding in this study. One qualitative study previously mentioned brokers in focus group discussions with community members about seeking general sexual reproductive healthcare ${ }^{7}$. Some focus group participants seemed to think that a broker was necessary in order to obtain a doctor's appointment and that it was not possible to access the doctor or obtain a hospital admission

Page $13 / 18$ 
directly ${ }^{7}$. The sentiment among some community members was that doctors in the government health center and brokers are working together. However, the MR providers in this study clearly see brokers as a barrier to accessing clinic MR services and believe that the brokers faciliate unsafe care for patients by taking them to informal clinics where the providers may not be trained and the facilities may not be clean or the equiptment sterile. There was no indication of collaboration between these trained MR providers and brokers, and many providers thought that the brokers should be punished.

\section{Limitations}

One limitation of this qualitative study is that it is not generalizable to all MR providers in Bangladesh. The providers interviewed work only in tertiary care settings within urban divisional capitals. Because of this, they often witness the most complicated MR cases, which may cause them to have a more negative opinion of unsanctioned providers, pharmacists, and self-management of abortion. Additionally, providers in this study have limited experience with unsanctioned providers and self-management of abortion when it happens safely without complications since those patients are not seen for their care. Other studies have noted higher complication rates with out-of-clinic abortion, though studies focusing on patients often describe their experiences with out-of-clinic providers and pharmacists favorably ${ }^{3,7}$. However, this may be due to the high satisfaction rates of abortion clients in general ${ }^{3,7}$. Another limitation of the current study is that, with the exception of the providers who have performed site visits to investigate unsanctioned clinics, most providers have not directly witnessed the conditions and quality of care of out-of-clinic providers/pharmacists and only know through patient report and word-of-mouth. With regards to qualitative methodology, one limitation is that the semi-structured interviews were not performed by the qualitative researchers responsible for the data coding and analysis, which would have been ideal. However, the study team felt like the interviews needed to be performed by individuals of a similar cultural background to increase cultural concordance, and interviews were performed by local professionals trained by the in-country team. To account for this, team coding was performed to promote discussion and consensus, addressing any discrepancies in code assignment or description. The qualitative researchers maintained contact with the in-country interviewers, providing feedback on interview and transcription techniques and asking clarifying questions of translations. Finally, there is little demographic information known about the providers that were interviewed, such as age, religion, and years in practice. Given the senstitive nature of the topic and potential stigma for providing care, little demographic information was collected to maintain confidentiality. However, provider responses may be impacted by confounding characteristics not recognized in this study.

Further studies are needed to provide a more robust picture of out-of-clinic and self-managed abortion in Bangladesh. Qualitative studies among providers in more rural or peripheral clinical settings would be beneficial to assess the quality of services, barriers and facilitators to improving MR services in these peripheral settings, and their experiences with complications. Qualitative studies with informal providers about MR/abortion care specifically would also be helpful in obtaining a more direct depiction of their services, their experiences with complications, and their willingness and capacity to receive further training. Finally, interviews with MR clients seeking care out-of-clinic would provide further insight into their abortion experiences and barriers/facilitators of out-of-clinic and self managed abortion, especially among clients who have not experienced complications. 
Despite the need for further studies, this study was unique in that it outlined provider perspectives of MR/PAC/abortion in-detail and gave insight in how to improve MR safety in Bangladesh.

\section{Conclusion}

There was a lack of consensus among trained providers about the definition of MR/abortion and gestational age cutoffs. Self-management of abortion using Misoprostol (with or without Mifepristone) from pharmacists, who do not provide adequate instruction, is increasing, while the use of traditional healers and foreign objects in the cervix or uterus is decreasing. Some unsanctioned providers use MVA and/or sharp curette to provide abortion services outside of clinic settings. Brokers intercept patients entering the hospitals/clinics and receive a commission from unsanctioned clinics for bringing patients, which creates barriers to safe MR care. Other barriers to accessing in-clinic MR include distance, family influence, lack of knowledge, and provider judgement. Providers in this study are located in urban tertiary care facilities and are mostly seeing the complicated MR/PAC cases, so the safety of out-of-clinic/self-managed abortion is unknown. Further studies among providers in more peripheral settings and MR clients who have attempted out-of-clinic abortions are needed.

\section{List Of Abbreviations}

Menstrual regulation (MR)

Post-abortion care (PAC)

Manual vaccum aspiration (MVA)

Dilation and curettage (D\&C)

Reproductive Health Services Training and Education Program (RHSTEP)

Non-governmental organization (NGO)

Semi-structured interviews (SSIs)

Family Welfare Visitors (FWVs)

\section{Declarations}

\section{Ethics approval and consent to participate}

Ethical approval of all study instruments and protocols was granted by the Bangladesh Medical Research Council (BMRC) and the University of California, San Diego Institutional Review Board. All participants gave signed informed consent to participate prior to being interviewed, including the consent to be audio recorded.

\section{Consent for publication}

Not applicable. 


\section{Availability of data and material}

Data sharing is not applicable to this article as no datasets were generated or analysed during the current study.

\section{Competing interests}

The authors have no conflicts of interest to report.

\section{Funding}

This study was funded by the Society of Family Planning, grant SFPRF18-10. These funds were used to hire and support local data collectors, data management, development of data collection materials, and researcher travel. The funder was involved in reviewing and approving the study design but was not involved in data collection, analysis, or manuscript development.

\section{Author's contributions}

All authors have read and approved the manuscript. BC is the study PI and developed the study protocol and all data collection materials and was responsible for the study activities. She supervised the data collection process and performed all data coding, analysis, and manuscript writing. EP trained the qualitative interviewers and provided insight on the study protocol and data collection materials. ST contributed to data coding and analysis. $\mathrm{SH}$ is a qualitative methods expert and provided insight in creating the data collection tools, data coding, analysis, and manuscript writing. DP is the local supervisor in Bangladesh and oversaw the data collection process. FA assisted in the data collection process and managed the transcription and translation of the interviews. JS and SA contributed to the study protocol, data collection tools, and manuscript writing.

\section{Acknowledgements}

The authors would like to thank the providers who gave their time to participate in this study. They would also like to thank BAPSA and Ipas, Bangladesh, who provided training to qualitative interviewers as well as transcription and translation of all interview transcripts and local oversight of all study activities. They would also like to thank the Society of Family Planning for funding this study.

\section{References}

1. Say L, Chou D, Gemmill A, Tuncalp O, Moller AB, Daniels J, Gulmezoglu AM, Temmerman M, Alkema L. Global causes of maternal death: a WHO systematic analysis. The Lancet Global Health. 2014;2(6):e323e333.

2. Guttmacher Institute: Menstrual Regulation and Induced Abortion in Bangladesh Fact Sheet. https://www.guttmacher.org/sites/default/files/factsheet/menstrual-regulation-unsafe-abortionbangladesh.pdf (2012). Accessed 15 June 2019. 
3. Hossain A, Moseson H, Raifman S, Gerdts C, Biswas KK, Greene Foster, D. How shall we survive: a qualitative study of women's experiences following denial of menstrual regulation services in Bangladesh. Reprod Health. 2016;13(1):86.

4. Singh S, Hossain A, Maddow-Zimet I, Bhuiyan U, Vlassoff M, Hussain R. The incidence of menstrual regulation procedures and abortion in Bangladesh, 2010. International Perspectives on Sexual and Reproductive Health. 2012;38(3):122-32.

5. Vlassoff M, Hossain A, Maddow-Zimet I, Singh S, Bhuiyan HU. Menstrual regulation and postabortion care in Bangladesh: factors associated with access to and quality of services. 2012.

https://www.guttmacher.org/sites/default/files/report_pdf/bangladesh-mr.pdf. Accessed 15 June 2019.

6. Rahman M, DaVanzo J, and Razzaque A. Pregnancy termination in Matlab, Bangladesh: maternal mortality risks associated with menstrual regulation and abortion. Int Perspect Sex Reprod Health. 2014;49(3):108-18.

7. Mahmud I, Chowdhury S, Siddiqi BA, Theobald S, Ormel H, Biswas S, Jahangir YT, Sarker M, Rashid SF. Exploring the context in which different close-to-community sexual and reproductive health service providers operate in Bangladesh: a qualitative study. Human Resources for Health. 2015; doi:10.1186/s12960-0150045-z.

8. Hsieh HF, Shannon SE. Three approaches to qualitative content analysis. Qualitative Health Research. 2005;15(9):1277-1288.

9. Gibson WJ, Brown A. Identifying themes, codes and hypotheses. In: Working with Qualitative Data. Thousand Oaks, CA: Sage Publications; 2009. p. 132-133.

10. Saldaña J. The Coding Manual for Qualitative Researchers. $2^{\text {nd }}$ Thousand Oaks, CA: Sage Publications; 2013.

11. MacQueen KM, McLellan-Lemal E, Bartholow K, Milstein B. Team-based codebook development: Structure, process, and agreement. In: Guest G and MacQueen KM, editors. Handbook for Team-based Qualitative Research. Lanham, MD: AltaMira Press. P. 119-135.

12. Dedoose Version0.35. SocioCultural Research Consultants, LLC, Los Angeles, CA. 2018. www.dedoose.com. Accessed 15 December 2018.

13. DaVanzo J and Rahman M. Pregnancy termination in Matlab, Bangladesh: trends and correlates of use of safer and less-safe methods. Int Perspect Sex Reprod Health. 2014;40(3):119-26.

14. Huda FA, NGO TD, Ahmed A, Alam A, Reichenbach L. Availability and provision of misoprostol and other medicines for menstrual regulation among pharmacies in Bangladesh via mystery client survey. Int $\mathrm{J}$ Gynacol Obstet. 2014;124(2):164-8.

15. Footman K, Scott R, Taleb F, Dijkerman S, Nuremowla S, Reiss K, Church K. Feasibility of assessing the safety and effectiveness of menstrual regulation medications purchased from pharmacies in Bangladesh: a prospective cohort study. Contraception. 2017; doi.10.1016/j.contraception.2017.08.002.

16. Huda FA, Mahmood HR, Alam A, Ahmmed F, Karim F, Sarker BK, Haque NA, Ahmed A. Provision of menstrual regulation with medication among pharmacies in three municipal districts of Bangladesh: a situation analysis. 2018;97:144-151.

17. Marlow HM, Biswas K, Griffin R, Menzel J. Women's experiences with medication for menstrual regulation in Bangladesh. Culture, Health \& Sexuality. 2016;18(3):349-360.

Page $17 / 18$ 
18. Government of Bangladesh. Family Planning 2020 Commitment. 2017.

https://www.familyplanning2020.org/sites/default/files/Govt_Bangladesh_FP2020_Commitment_2017.pdf. Accessed 12 May 2019.

19. Jain A, Hardee K. Revising the FP Quality of Care Framework in the Context of Rights-based Family Planning. Studies in Family Planning. 2018;49(2):171-179.

20. Yasmin R, Rob U, Hena IA, Das TR, Ahmed FU. Increasing access to safe menstrual regulation services in Bangladesh by offering medical menstrual regulation. Reprod Health Matters 2015;22 Suppl 1:67-74.

\section{Supplementary Files}

This is a list of supplementary files associated with this preprint. Click to download.

- COREQchecklistBangladeshStudy.pdf

- BangladeshQualitativeQuestionsProviders.docx 